\title{
Research of the Applicability of the Active Laser Turbulent-degraded Image Recognition Algorithm
}

\author{
Yao Zhang ${ }^{1, a}$, Xiaoya Liu ${ }^{2, b}$ \\ ${ }^{1}$ Scool of Computer and Communication Engineering \\ Northeastern University at Qinhuangdao, Qinhuangdao 066004, Hebei, China \\ ${ }^{2}$ University of lowa, lowa City, IA, U.S.A

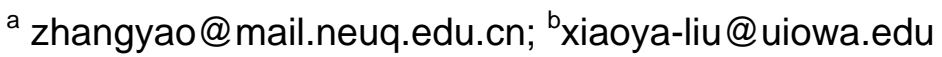

Keywords: Scintillation effect of turbulence; Active Laser; Sub-pixel; High-temperature environment

\begin{abstract}
The Scintillation effect of turbulence on laser measurement in high temperature environment will cause light moves, bend, or shake according to the ideal path. The object will have fuzzy dispersion and abnormal image on focal plane. By measuring the thickness of the highenvironment glass, using characteristics of the distribution of the linear array CCD image spot inner-pixel grayscale in high-temperature environment and high relevance between each image spot, to create a mathematic model which can report the relationship between grayscale of the target area and the location of the center of mass of the target image spot as accurately as possible. By using this model, the accurate estimation of the location of the target point will be achieved.
\end{abstract}

\section{Introduction}

Using CCD to probe the accurate location of the long-distance target or close target point is widely applied ${ }^{[1][2]}$. CCD image spot subdivision method basically uses the characteristic of the grayscale distribution of the spot and subdivision algorithm to find the location of the target image spot, which can make the measurement accuracy to reach sub-pixel level ${ }^{[3][4]}$. In uniform index of reflection medium without considering aberration, object will image ideally, which means object is completely similar to the image. In high-temperature environment, the distribution of the gas index of reflection will change due to some reason such as the changing of the distribution of the gas temperature and gas flow. Turbulent flash effect on laser transmission can directly cause the distortion, blur, and deterioration of the image, which increase the difficulty of the accurate image spot locating. There are two classic simulation research methods at present: Frequency domain simulation and direct simulation of the spatial domain. Frequency domain simulation can get space random phase shift according from air turbulent power spectral density function (PSDF). Then by doing Fourier transform, frequency domain simulation will acquire distribution of two-dimensional space random phase shift, which is known as Fast Fourier Transform (FFT). Direct simulation of the spatial domain can get space random phase shift from a random linear set from orthonormal complete basis function. Zernike Polynomials is one of the common set from orthonormal complete basis function ${ }^{[5]}$. By measuring the thickness of the high-environment glass, using characteristics of the distribution of the linear array CCD image spot inner-pixel grayscale in high-temperature environment and high relevance between each image spot, to do the research of the applicability of the active laser turbulent-degraded image recognition algorithm.

\section{The Scintillation effect of turbulence}

The frequency of gas flash is commonly under $1000 \mathrm{~Hz}$. Peak frequency can stand between $10 \sim 100 \mathrm{~Hz}$. Relative theory and experimental research mainly focus on the flicker index light inter undulation variance, which is basically shows as:

$$
\sigma_{I}^{2}=<I^{2}>/<I>^{2}-1
$$


In this formula, I is stand for light intensity, <> is stand for average of the system.

Mostly, logarithmic canonical distribution is the gas flicker probability distribution when single beam pass through weak fluctuation turbulent gas. Light intensity fluctuation can be predicted nicely by using Rytov approximation. Assume the amplitude and phase of actual practical electromagnetic wave component $u$ are $\mathrm{A}$ and $\mathrm{S}$, and the amplitude and phase of non-disturbance wave component $u_{0}$ of $u$ are $A_{0}$ and $S_{0}$, there is:

$$
\begin{aligned}
& u=A \exp (j S) \\
& u_{0}=A_{0} \exp \left(j S_{0}\right)
\end{aligned}
$$

Express logarithmic amplitude undulation ${ }^{x}$ as the sum of large amount of independent forward scatters. According to the central limit theorem, ${ }^{x}$ satisfies normal distribution. By using the former part of analysis, it is known that lognormal distribution only usable at weak fluctuation turbulent gas area at $\sigma_{x}^{2}<0.2 \sim 0.5$, and it will show variance saturated flicker area when transmitting in strong fluctuation turbulent gas or doing long-distance transmission[6][7].

Under the condition of weak fluctuation turbulent flow gas, assume the average of logarithmic amplitude is $\langle x\rangle$ and the variance is $\sigma_{x}^{2}$, so:

$$
P_{A}(A)=\frac{1}{\sqrt{2 \pi} \sigma_{x}} \exp \left\{-\frac{(x-<x>)^{2}}{2 \sigma_{x}^{2}}\right\}
$$

In order to get the probability density function of amplitude $A=A_{0} \exp (x)$, probability transform is introduced:

$$
P_{A}(A)=P_{x}\left(x=\ln \left(A / A_{0}\right)\right)\left|\frac{d x}{d A}\right|=P_{x}\left(x=\ln \left(A / A_{0}\right)\right) \frac{1}{A}
$$

Also:

$$
P_{A}(A)=\frac{1}{\sqrt{2 \pi} \sigma_{x} A} \exp \left\{-\frac{\left(\ln \left(A / A_{0}\right)-<x>\right)^{2}}{2 \sigma_{x}^{2}}\right\} \quad A \geq 0
$$

Similarly, the probability density function of $I=A^{2}$ can be:

$$
P_{I}(I)=\frac{1}{2 \sqrt{2 \pi} \sigma_{x} I} \exp \left\{-\frac{\left(\ln \left(I / I_{0}\right)-2<x>\right)^{2}}{8 \sigma_{x}^{2}}\right\} \quad I \geq 0
$$

In actual turbulent gas, gas flicker is considered as a modulation where small-size turbulent flicker act on large-size turbulent flicker. Due to the random modulation of receiving light intensity cause by the beam jitter of the large-size turbulent flow, the average of receiving light intensity $<I>$ is a random value.

By doing a lot of research on using linear array CDD scanning single image spot sequence in high-temperature lab environment, as shown in figure1 and figure 2, image-dealing sub-pixel algorithm can be found which is focus on its characteristic of the optical system.

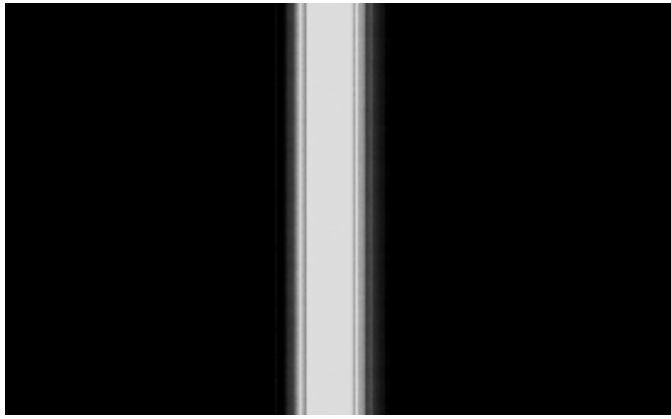

Figure 1, Single spot scanned in the lab
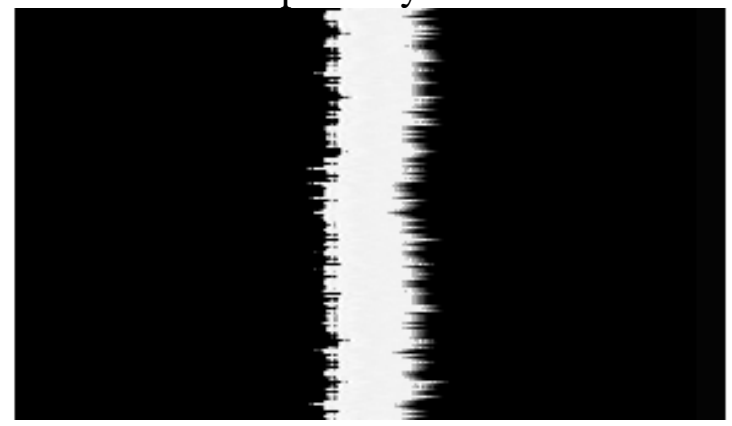

Figure 2, Single spot scanned in hightemperature production environment 


\section{Method of Weighted polynomial difference}

According to the theorem of polynomial difference:

$$
\begin{aligned}
& f(x)=\sum_{k=0}^{k=n} \frac{\omega_{n+1}(x)}{\left(x-x_{k}\right) \omega_{n+1}^{\prime}\left(x_{k}\right)}=\sum_{k=0}^{k=n} l_{k}(x) f\left(k_{x}\right) \\
& \quad \omega_{n+1}(x)=\prod_{m=0}^{m=n}\left(x-x_{m}\right) \quad \omega_{n+1}^{\prime}\left(x_{k}\right)=\prod_{m=0}^{m=n}\left(x_{k}-x_{m}\right), x_{k} \text { are difference node. } f\left(x_{k}\right) \text { is function }
\end{aligned}
$$
value. $l_{k}(x)$ is basis function. $f(x)$ is the needed difference function.

Assume after-filtering video signals $\left(x_{0}, y_{0}\right),\left(x_{0}, y_{0}\right) \ldots\left(x_{n}, y_{n}\right)$. When beam spot comes to ${ }^{x_{j}}$, set $\left\langle x_{j-1}, x_{j+1}>,<x_{j-2}, x_{j+2}>\ldots<x_{j-m}, x_{j+m}>\right.$ as the interpolation nodes. Under this situation, $m=\min (j, n-j) .\left\langle x_{k 1}, x_{k 2}>\right.$ means setting $x_{k 1}$ and $x_{k 2}$ as the interpolation nodes and doing interpolation with $y_{k 1}, y_{j}$ and $y_{k 2}$ as function value. By doing this process, there are $m$ number of beam spots location can be found. Average these values and new beam spot location can be acquired:

$$
\left.\begin{array}{l}
x_{p 1}=x_{j}+\frac{1\left(y_{j-1}-y_{j+1}\right)}{2\left(y_{j-1}-2 y_{j}+y_{j+1}\right)} \\
x_{p 2}=x_{j}+\frac{2\left(y_{j-2}-y_{j+2}\right)}{2\left(y_{j-2}-2 y_{j}+y_{j+2}\right)} \\
x_{p m}=x_{j}+\frac{m\left(y_{j-m}-y_{j+m}\right)}{2\left(y_{j-m}-2 y_{j}+y_{j+m}\right)}
\end{array}\right\} \Rightarrow x_{p}=\frac{\sum_{i=1}^{i=m} x_{p i}}{m}
$$

The algorithm above is doing average among $m$ beam spots. The beam spot location of each $^{m}$ spots has the same contribution towards the result of the locating. But experimentally, the closer the interpolation nodes close to ${ }^{X_{j}}$ when beam are set at ${ }^{X_{j}}$, the more the result contribute towards the locating results. In other way, the sequence of the location after interpolation $\left(x_{p 1}, x_{p 2}, \ldots, x_{p m}\right)$ is listed as the decreasing contribution of the locating results. Therefore, using weighted average algorithm is more reasonable. A simple way to determine the weights is following these instructions: the weights getting from the sequence formed by distance between the interpolation nodes and $x_{j}$ followed by $(m, m-1, \ldots, 1)$. Then naturalize the weights and get $\left(p_{1}, p_{2}, \ldots, p_{m}\right)$.Get:

$$
P_{k}=\frac{(m+1-k)}{\sum_{i=1}^{i=m} i} \quad(1 \leq k \leq m)
$$

The final location of the beam spot is:

$$
x=\sum_{i=1}^{i=m} x_{p k} p_{k}
$$

\section{Method of the multi-threshold}

Use method of the proportion of multi-threshold center act as the Sub-pixel subdivision positioning algorithm. Method of the proportion of multi-threshold center combines the advantages of laddering technique and Proportional Centre Act. Choose multiple proper thresholds $V_{n}$ and intersect with graph at $p_{n}$ and $q_{n}$. Find the location $b_{n 1}, b_{n 2}, b_{n 3}, b_{n 4}$ and grey scale value 
$m_{n 1}, m_{n 2}, m_{n 3}, m_{n 4}$ where the point are adjacent to the intersect point. Using the interpolation formulas below can calculate the location of $a_{n}, a_{n}$ where $p_{n}$ and $q_{n}$ are corresponded. Interpolation formulas are:

$$
\begin{aligned}
& a_{n 1}=b_{n 1}-\frac{\left(b_{n 2}-b_{n 1}\right)\left(m_{n 2}-V_{n}\right)}{m_{n 2}-m_{n 1}} \\
& a_{n 2}=b_{n 3}-\frac{\left(b_{n 3}-b_{n 4}\right)\left(m_{n 3}-V_{n}\right)}{m_{n 3}-m_{n 4}}
\end{aligned}
$$

Therefore the center of the image is located at:

$$
a_{n}=\frac{a_{n 1}+a_{n 2}}{2}
$$

\section{Experiment Result:}

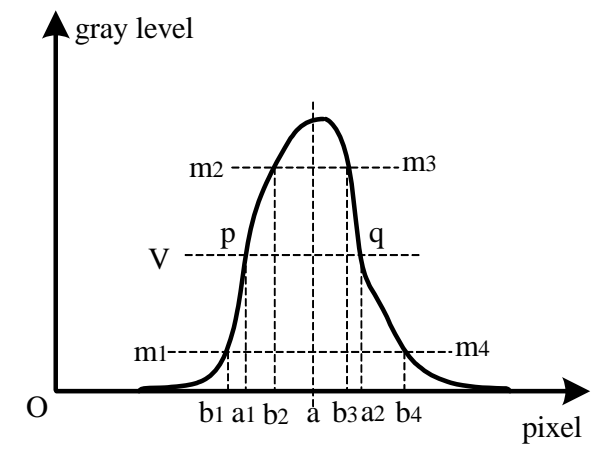

Figure 3 Principle diagram of Method of the

Using different pixel subdivision algorithm measure the thickness of a piece of standard glass sample (standard thickness is $7.84 \mathrm{~mm})$ in high-temperature environment $\left(650^{\circ} \mathrm{C}\right)$. Data are shown in table 4:

\begin{tabular}{|l|c|c|c|c|c|c|c|c|c|}
\hline order number & 01 & 02 & 03 & 04 & 05 & $\ldots$ & 98 & 99 & 100 \\
\hline stem $Z_{j}$ & 7.842 & 7.837 & 7.839 & 7.840 & 7.831 & $\ldots$ & 7.838 & 7.845 & 7.847 \\
\hline Multi threshold $^{J_{j}}$ & 7.841 & 7.838 & 7.835 & 7.842 & 7.833 & $\ldots$ & 7.841 & 7.839 & 7.835 \\
\hline $\begin{array}{l}\text { Weighted } \\
\text { approximation } K_{j}\end{array}$ & 7.838 & 7.840 & 7.841 & 7.840 & 7.835 & $\ldots$ & 7.843 & 7.837 & 7.842 \\
\hline
\end{tabular}

Table 4 Data collect from calculation using different pixel subdivision algorithm

The experiment shows that the standard deviation of method of center of mass is $\sigma_{Z}=0.0054508 \mathrm{~mm}$; Standard deviation of method of the proportion of multi-threshold center act is $\sigma_{\mathrm{J}}=0.003091 \mathrm{~mm}$; Standard deviation of method of weighted polynomial difference is

$\sigma_{\mathrm{k}}=0.0027682 \mathrm{~mm}$.

\section{Conclusion}

According from the theoretical analysis and experiment test, using the method of calculating the interpolation for nodes around the light spot, which is method of weighted polynomial difference, can get the lowest standard deviation $\sigma_{\mathrm{k}}=0.0027682 \mathrm{~mm}$. This method of analysis can increase the stability of the center of the light spot; but it has disadvantages such as the algorithms are relatively complex, occupy more system resources, which will affect the speed of measuring in the production line.

Although method of the proportion of multi-threshold center act has less accuracy than method of weighted polynomial difference, it combines the advantages of laddering technique and Proportional Centre Act. CCD signals can overcome the problem of waveform moving around and swinging pointer-like. Theoretically, method of the proportion of multi-threshold center act can do subdivision on CCD pixel without limit. According to the experiment and practical application in the production line, static resolution of the measurement system is $1 \mu \mathrm{m}$ when the thickness of glass is changing within $2 \sim 15 \mathrm{~mm}$. The uncertainty of the measuring is $7 \mu \mathrm{m}$. As a result, the need of 
measuring of sub-pixel active laser turbulent-degraded can be completely satisfy by using the CCD sub-pixel subdivision algorithm form method of the proportion of multi-threshold center act.

\section{References}

[1] LANG B,DONG M L,WANG J. Sub-pixel location of center of target based on Zernike moment[J].SPIE,2010,7544:75443A-1-75443A-6.

[2] JIA H,YANG J K,LI X J.Minimum variance unbiased sub-pixel centroid estimationof point image limited by photon shot noise[J].Opt.Soc.Am.A,2010,27(9):2038-204 5.

[3] CHEN Y J,FENG Y,WEI L A. Experiment research on sub-pixel location error of thefaculacentroid [J]. Opto-Electronic Engineering, 2010, 37(2):80-84. (in Chinese)

[4] ZHANG B N,AN H M,MA0 CH L.Study on Displacement Sensor Based on Difference 0peration Spot Center Location Algorithm [J].Chinese Journal of Sensors and Actuators, 2011,24(2):215-219.(in Chinese)

[5] CARBILLET M,RICCARDI A.Numerical modeling of atmospherically perturbed phase screens: new solutions for classical fast Fourier transform and Zernike methods [J].App1.Optics, 2010, 49(31):47-52. 